\title{
The Finite Element Method for Infinite Domains. I
}

\author{
By Ivo Babuška*
}

\begin{abstract}
Numerical methods (finite element methods) for the approximate solution of elliptic partial differential equations on unbounded domains are considered, and error bounds, with respect to the number of unknowns which have to be determined, are proven.
\end{abstract}

1. Introduction. The finite element method, its theory and practice, has recently become of interest in numerical analysis, see e.g. [1] [13], and the papers of Aubin, Birkhoff, Bramble, Ciarlett, Schatz, Schultz, Varga, etc.

The theoretical analyses of the finite element method have been concerned with bounded domains. Strang and Fix, [1], [2], have, however, analyzed the finite element method with respect to an infinite domain (the space $R_{n}$ ), but their procedure requires the solution of an infinite system of linear algebraic equations.

This paper will deal with the problem of finding, by the finite element method, an approximate solution of a boundary value problem for elliptic partial differential equations on an infinite domain by solving only a finite system of linear algebraic equations.

The approach will be shown on a model problem. Our task will be to find the solution of the equation (weak solution)

$$
-\Delta u+u=f
$$

on $R_{n}$, where $u \in W_{2}^{1}\left(R_{n}\right)$ and $f \in W_{2}^{k}\left(R_{n}\right), k \geqq 0$.

We will show that the rate of convergence on compact sets of $R_{n}$ is practically the same as the rate of convergence for boundary value problems on bounded domains. The rate of convergence will turn out to be determined by the number of unknowns in the system of linear algebraic equations.

Our approach may be easily generalized to the case of an elliptic differential equation of order $2 \mathrm{~m}$, provided that the coefficient of the zero order term of the equation is bounded above and below by positive constants.

We will analyze only the case when $\Omega=R_{n}$. By combining the approach described above with the results concerning bounded domains (see e.g. [6] [11]), it is easy to get the corresponding results for unbounded domains with bounded boundary.

Throughout this paper, let $x$ denote the $n$-dimensional vector in $R_{n}$, i.e. $x \equiv$ $\left(x_{1}, \cdots, x_{m}\right)$ where $x_{i} \in R_{1}, i=1,2, \cdots, n$.

Received November 2, 1970, revised April 21, 1971.

AMS 1969 subject classifications. Primary 3504; Secondary 6566.

Key words and phrases. Numerical element method, numerical method for elliptic equations and unbounded domains.

* This research was supported in part by the National Science Foundation under Grant No. NSF GU-2061 and in part by the Atomic Energy Commission under Contract No. AEC AT-(40-1) $3443 / 3$. 
Let $\|x\|^{2}=\sum_{i=1}^{n} x_{i}^{2}$ and $|x|=\sum_{i=1}^{n}\left|x_{i}\right|$. If $a \in R_{1}$, let $x+a_{i} \equiv\left(x_{1}, \cdots, x_{i-1}\right.$, $\left.x_{i}+a, x_{i+1}, \cdots, x_{n}\right)$. Let $k$ denote a multi-integer, i.e. $k \equiv\left(k_{1}, \cdots, k_{n}\right)$, where $k_{i}$ is an integer, $i=1,2, \cdots, n$. Let $\|k\|^{2}=\sum_{i=1}^{n} k_{i}^{2}$ and $|k|=\sum_{i=1}^{n}\left|k_{i}\right|$. Define the inequality $k \geqq 0$ to mean $k_{i} \geqq 0, i=1,2, \cdots, n$. Let $C$ denote a generic constant which may have different values wherever it appears in the text.

2. The Spaces. In this section, we shall introduce the spaces which will be used in the paper.

Definition 2.1. Let the space $W_{2, \mu}^{l}\left(R_{n}\right)$, with $l \geqq 0$ an integer, $\mu$ real, be the Banach space of all functions $u$ such that

$$
\|u\|_{W_{1, \mu^{2}\left(R_{n}\right)}^{2}}=\int_{R_{n}} e^{2 \mu|x|} \sum_{|k| \leq l_{i k} \geq 0}\left(D^{k} u\right)^{2} d x<\infty,
$$

where $D^{k}=\partial^{k_{1}+k_{9}+\cdots+k_{n}} / \partial x_{1}^{k_{1}} \cdots \partial x_{n}^{k_{n}}$. For $\mu=0$, we get the usual Sobolev space. For $\mu \neq 0$, we get a weighted Sobolev space. Note that $L_{2}\left(R_{n}\right)=W_{2,0}^{0}\left(R_{n}\right)$.

Let us now introduce the so called $B$ splines. For $x \in R_{1}$, let

$$
\begin{aligned}
\varphi_{1}(x) & =1, & |x|<\frac{1}{2}, \\
& =0, & |x| \geqq \frac{1}{2} .
\end{aligned}
$$

Let $t$ be an integer $\geqq 2$. Starting with $t=2$, we recursively define $\varphi_{t}(x)$ as

$$
\varphi_{t}(x)=\varphi_{1}(x) * \varphi_{t-1}(x), \quad t \geqq 2,
$$

where * denotes convolution.

Now, for $x \in R_{n}, x \equiv\left(x_{1}, \cdots, x_{n}\right)$, define

$$
\varphi_{t}(x)=\prod_{j=1}^{n} \varphi_{t}\left(x_{i}\right), \quad t \geqq 1,
$$

and

$$
\varphi_{i, i}(x)=\varphi_{t-1}\left(x_{i}-\frac{1}{2}\right) \prod_{i=1 ; j ; i}^{n} \varphi_{i}\left(x_{i}\right), \quad t \geqq 2, t=1, \cdots, n .
$$

Let us mention some of the well-known properties of these functions which will be important later on:

(1) $\varphi_{t}(x) \geqq 0, \varphi_{t, i}(x) \geqq 0$ for all $x \in R_{n s}$

(2) $\varphi_{t}(x)$ and $\varphi_{t, i}(x)$ have compact support.

(3) Denoting by $F(\varphi)(\sigma)$, the Fourier transform of $\varphi(x)$, we have, with $\sigma=\left(\sigma_{1}, \cdots, \sigma_{n}\right)$,

$$
\begin{gathered}
F\left(\varphi_{t}\right)(\sigma)=\prod_{i=1}^{n}\left(\frac{\sin \frac{1}{2} \sigma_{i}}{\frac{1}{2} \sigma_{i}}\right)^{t}, \\
F\left(\varphi_{i, i}\right)(\sigma)=\left(\frac{\sin \frac{1}{2} \sigma_{i}}{\frac{1}{2} \sigma_{i}}\right)^{i-1} e^{i \sigma i / 2} \prod_{i=1 ; i, i}^{n}\left(\frac{\sin \frac{1}{2} \sigma_{i}}{\frac{1}{2} \sigma_{i}}\right)^{\prime}, \\
\frac{\partial \varphi_{t}(x)}{\partial x_{i}}=-\varphi_{i, i}(x)+\varphi_{i, i}\left(x+1_{i}\right) .
\end{gathered}
$$

In the following, let $U$ and $V$ denote the functions defined on the set of all multiintegers $k$. 
Definition 2.2. Let the space $Q_{\mu}^{l, n}, l=0,1, h>0, \mu$ real, be the space of all functions $U$ such that

$$
\|U\|_{0_{k} \cdots A}^{2}=h^{n} \sum_{k}|U(k)|^{2} e^{2 \mu k|k|}<\infty
$$

(for $l=0$ ) and

$$
\|U\|_{\alpha_{k}^{2}, 1}^{2}=h^{n}\left[\frac{1}{h^{2}} \sum_{k} \sum_{i=1}^{n}\left|U\left(k+1_{i}\right)-U(k)\right|^{2} e^{2 \mu k|k|}+\sum_{k}|U(k)|^{2} e^{2 \mu \lambda|k|}\right]<\infty
$$

(for $l=1$ ).

Let $U \in Q_{\mu}^{l, k}$ for $l=0$ and $l=1$. Define the mapping $S_{i}^{\star}, j \geqq 1$, by

$$
S_{i}^{h} U=\sum_{k} U(k) \varphi_{i}(x / h-k),
$$

and the mapping $S_{i}^{h, i}, j \geqq 2$, by

$$
S_{i}^{h, i} U=\sum_{k} U(k) \varphi_{i, i}(x / h-k) .
$$

Throrem 2.1. For $|\mu|$ sufficiently small, there exist constants $C_{1}$ and $C_{2}, 0<C_{1}<$ $C_{2}<\infty$, such that

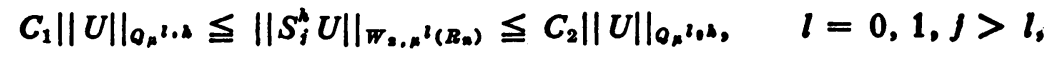

and

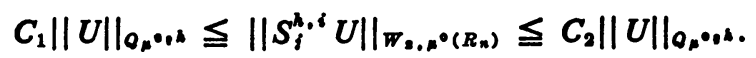

Proof. 1. Let us first consider (2.13) for $l=0$ and $\mu=0$. It is sufficient to prove this inequality for $h=1$.

In [14] we have proved that

$$
F\left(S_{i}^{1} U\right)(\sigma)=Z_{i}(\sigma) \sum_{k} U(k) \exp (i\langle k, \sigma\rangle),
$$

where

$$
Z_{i}(\sigma)=F\left(\varphi_{i}\right)(\sigma)
$$

and

$$
\langle k, \sigma\rangle=\sum_{i=1}^{n} k_{i} \sigma_{i}
$$

By a well-known property of the Fourier transform, we have

$$
\left\|F\left(S_{j}^{1} U\right)\right\|_{L,\left(R_{n}\right)}^{2}=(2 \pi)^{n}\left\|S_{i}^{1} U\right\|_{L,\left(R_{*}\right)}^{2} .
$$

Hence, we may write

$$
\left\|F\left(S_{i}^{1} U\right)\right\|_{L_{0}\left(B_{n}\right)}^{2}=\sum_{l} \int_{\Omega_{0}}\left|\sum_{k} U(k) \exp (i\langle k, \sigma\rangle)\right|^{2}\left|Z_{j}(\sigma-l)\right|^{2} d \sigma,
$$

where $\Omega_{0}=\left\{x ;\left|x_{i}\right|<\pi\right\}$. Since there exists a $C$ such that $\left|Z_{i}(\sigma)\right|^{2}>C>0$ on $\Omega_{0}$, we have, from (2.19), 
(2.20) $\left\|F\left(S_{i}^{1} U\right)\right\|_{L_{3}\left(R_{n}\right)}^{2} \geqq C \int_{\Omega_{0}}\left|\sum_{k} U(k) \exp (i\langle k, \sigma\rangle)\right|^{2} d \sigma \geqq C(2 \pi)^{n} \sum_{k}|U(k)|^{2}$

From (2.20), (2.18) and (2.9), we obtain

$$
\left\|S_{i}^{1} U\right\|_{L_{2}\left(R_{n}\right)} \geqq C\|U\|_{Q_{0} \cdot,_{1}} .
$$

From (2.19), we also have

$$
\left\|F\left(S_{i}^{1} U\right)\right\|_{L,\left(R_{n}\right)}^{2} \leqq \int_{\Omega_{0}}\left|\sum_{k} U(k) \exp (i\langle k, \sigma\rangle)\right|^{2} d \sigma \sum_{l} Z_{i, l}^{2}
$$

with

$$
Z_{i, l}=\max _{\sigma \in \Omega_{\bullet}}\left|Z_{i}(\sigma-l)\right| .
$$

From (2.6) it is clear that $\sum_{l} Z_{i, l}^{2}<\infty$. Therefore,

$$
\left\|S_{i}^{1} U\right\|_{L_{2}\left(R_{n}\right)} \leqq C\|U\|_{0, \cdots .1} \text {. }
$$

Inequalities (2.21) and (2.24) together prove (2.13) for the case $l=0$ and $\mu=0$. Inequality (2.14) can be proved in the same manner.

2. Let us now prove (2.13) for $l=0$ and $\mu \neq 0$. Let $U \in Q_{\mu}^{0,1}$ and $U_{\mu}(k)=U(k) e^{\mu|k|}$. Using (2.13) for $\mu=0$ and $U_{\mu}$, we obtain, because of (2.9),

$$
\int_{R_{n}}\left|\sum_{k}\right| U(k)\left|e^{\mu|k|} \varphi_{j}(x-k)\right|^{2} d x \leqq C\|U\|_{\beta_{\beta}^{2} \cdot 2 .}^{2}
$$

On the other hand, we have

$$
\left\|S_{i}^{1} U\right\|_{W, \mu^{\circ}\left(R_{n}\right)}^{2}=\int_{R_{n}}\left|\sum_{k} U(k) \varphi_{i}(x-k)\right|^{2} e^{2 \mu|x|} d x .
$$

Now since $\varphi_{i}(x) \geqq 0$ for all $x \in R_{n}$ and since $\varphi_{i}$ has compact support, it follows from (2.26) that

$$
\begin{aligned}
\left\|S_{i}^{1} U\right\|_{W_{3} \ldots \mu^{\circ}\left(R_{n}\right)} & \leqq \int_{R_{n}}\left|\sum_{k}\right| U(k)\left|\varphi_{i}(x-k)\right|^{2} e^{2 \mu|x|} d x \\
& \leqq\left. C \int_{R_{n}}\left|\sum_{k}\right| U(k)_{1} e^{\mu|k|} \varphi_{i}(x-k)\right|^{2} d x .
\end{aligned}
$$

From (2.25) and (2.27) we obtain

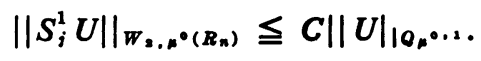

Now from (2.13) for $\mu=0$, we have with $C>0$, that

$$
\begin{aligned}
C\|U\|_{O_{\mu} \cdot 2}^{2} & \leqq C \int_{R_{n}}\left|\sum_{k}\right| U(k)\left|e^{\mu|k|} \varphi_{j}(x-k)\right|^{2} d x \\
& \leqq \int_{R_{n}}\left|\sum_{k} U(k) e^{\mu|k|} \varphi_{j}(x-k)\right|^{2} d x \\
& =\int_{R_{n}}\left|\sum_{k}\left[U(k) e^{\mu|x|} \varphi_{j}(x-k)+U(k)\left(e^{\mu|k|}-e^{\mu|x|}\right) \varphi_{j}(x-k)\right]\right|^{2} d x \\
& \leqq 2\left[\left\|S_{i}^{1} U\right\|_{W, \ldots \mu^{\circ}\left(R_{n}\right)}^{2}+\mu^{2} \int_{R_{n}}\left|\sum_{k}\right| U(k)\left|e^{\mu|k|} Q_{k}(x) \varphi_{i}(x-k)\right|^{2} d x\right],
\end{aligned}
$$


where $\left|Q_{k}(x)\right| \leqq C$, independently of $k$. Hence, we have

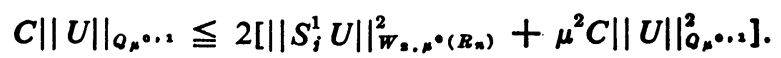

Therefore,

$$
\|U\|_{O_{\mu} \cdot 12} \leqq C\left\|S_{i}^{1} U\right\|_{W_{1, N^{\circ}\left(B_{n}\right)},}
$$

for sufficiently small $|\mu|$.

This proves (2.13) for the case $l=0$ and $\mu \neq 0, \mu$ sufficiently small in absolute , value. Inequality (2.14) can be proved for the case $l=0$ and $\mu \neq 0, \mu$ sufficiently small in absolute value, in the same manner.

3. Let us now prove the theorem for $l=1$. We have

$$
\begin{aligned}
\frac{\partial S_{i}^{h} U}{\partial x_{s}} & =\frac{1}{h}\left[-\sum_{k} U(k)\left[\varphi_{i, .}\left(\frac{x}{h}-k\right)-\varphi_{i . .}\left(\frac{x}{h}-1 .-k\right)\right]\right] \\
& =\frac{1}{h}\left[\sum_{k}(U(k+1 .)-U(k)) \varphi_{i . .}\left(\frac{x}{h}-k\right)\right] \\
& =\sum_{k} V(k) \varphi_{i, .}\left(\frac{x}{h}-k\right) .
\end{aligned}
$$

where

$$
V(k)=(U(k+1,)-U(k)) / h .
$$

From (2.14) with $l=0$, we obtain

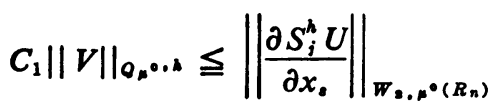

$$
\begin{aligned}
& \leqq C_{2}\|V\| \|_{\left.Q_{n} \cdots R_{n}\right)} \text {. }
\end{aligned}
$$

Inequality (2.14) follows immediately from (2.30), (2.31) and (2.32). This completes the proof of Theorem 2.1.

3. Some Auxiliary Theorems about a Bilinear Form. Let $\mathfrak{H C}_{n}=W_{2.4}^{1}\left(R_{n}\right) \times$ $W_{2,-\mu}^{1}\left(R_{n}\right)$. Define on $\mathcal{K}_{\mu}$ the bilinear form $A$ by

$$
A(u, v)=\int_{R_{n}}\left(\sum_{i=1}^{n} \frac{\partial u}{\partial x_{i}} \frac{\partial v}{\partial x_{i}}+u v\right) d x .
$$

We shall now prove some important properties of this bilinear form.

THEOREM 3.1. For sufficiently small $|\mu|$,

$$
\begin{aligned}
& |A(u, v)| \leqq C|| u\left\|_{w_{2, \mu^{2}\left(R_{*}\right)}}\right\| v \|_{W_{\mathrm{s},-\mu^{2}\left(R_{n}\right)},} \\
& \sup _{\|u\| W_{W_{2}, \mu^{2}\left(R_{n}\right) \leq 1}}|A(u, v)| \geqq C\|v\|_{\left.W_{2,-\mu^{2}\left(R_{n}\right)}\right)}
\end{aligned}
$$

where $C>0$ and

$$
\sup _{\|1\| \| W_{s,-\mu^{2}\left(R_{s}\right) \leq 1}}|A(u, v)| \geqq C\|u\|_{w_{B}, \mu^{2}\left(R_{n}\right)}, \quad C>0 .
$$

Proof. 1. Inequality (3.2) is easily proved by the following inequalities: 


$$
\begin{aligned}
& |A(u, v)|=\left|\int_{R_{n}}\left(\sum_{i=1}^{n} e^{\mu|x|} \frac{\partial u}{\partial x_{i}} e^{-\mu|x|} \frac{\partial v}{\partial x_{i}}+e^{\mu|x|} u e^{-\mu|x|} v\right) d x\right| \\
& \leqq \sum_{i=1}^{n}\left[\int_{R_{n}}\left(e^{\mu|x|} \frac{\partial u}{\partial x_{i}}\right)^{2} d x\right]^{1 / 2}\left[\int_{R_{n}}\left(e^{-\mu|x|} \frac{\partial v}{\partial x_{i}}\right)^{2} d x\right]^{1 / 2} \\
& +\left[\int_{R_{n}}\left(e^{\mu|x|} u\right)^{2} d x\right]^{1 / 2}\left[\int_{R_{n}}\left(e^{-\mu|x|} v\right)^{2} d x\right]^{1 / 2}
\end{aligned}
$$

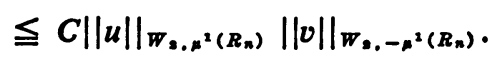

2. Let $\delta \in W_{2,-\mu}^{1}\left(R_{n}\right)$ and let $u=v e^{-2 \mu|x|}$. By direct computation, we have

$$
\frac{\partial\left(v e^{-2 \mu|x|}\right)}{\partial x_{i}}=e^{-2 \mu|x|} \frac{\partial v}{\partial x_{i}}-2 \mu e^{-2 \mu|x|} v \operatorname{sgn} x_{i} .
$$

Hence,

$$
\begin{aligned}
& \|u\|_{W_{1, \mu^{2}\left(R_{n}\right)}^{2}} \\
& \quad \leqq 2\left[\int_{R_{n}}\left[\sum_{i=1}^{n} e^{-4 \mu|x|}\left(\frac{\partial v}{\partial x_{i}}\right)^{2}+4 n \mu^{2} v^{2} e^{-4 \mu|x|}+v^{2} e^{-4 \mu|x|}\right] e^{2 \mu|x|}\right] d x \\
& \quad \leqq C\|v\|_{W=,-\mu^{2}\left(R_{n}\right)}^{2}
\end{aligned}
$$

which implies that $u \in W_{2, \mu}^{1}\left(R_{n}\right)$. Furthermore,

$$
\begin{aligned}
& \left|A\left(v e^{-2 \mu|x|}, v\right)\right| \\
& =\left|\int_{R_{n}} \sum_{i=1}^{n}\left[\frac{\partial v}{\partial x_{i}}-2 \mu v \operatorname{sgn} x_{i}\right]\left[\frac{\partial v}{\partial x_{i}}\right] e^{-2 \mu|x|} d x+\int_{R_{n}} v^{2} e^{-2 \mu|x|} d x\right| \\
& \geqq\|v\|_{W_{1},-n^{2}\left(R_{n}\right)}^{2}-2 \mu n\|v\|_{W_{1},-n^{2}\left(R_{n}\right)}^{2} \geqq C\|v\|_{W_{1},-n^{2}\left(R_{n}\right)}^{2} \text {. }
\end{aligned}
$$

Inequality (3.8), together with (3.7), proves (3.3).

3. Replacing $\mu$ by $-\mu$ in the above discussion we have inequality (3.4).

Let us prove a further theorem.

THEOREM 3.2. For sufficiently small $|\mu|$, we have

$$
\begin{aligned}
& \left|A\left(S_{i}^{h} U, S_{i}^{h} V\right)\right| \leqq C|| S_{i}^{h} U\left\|_{W_{1, \mu^{2}\left(R_{n}\right)}}\right\| S_{i}^{h} V \|_{\left.W_{2,-\mu^{2}\left(B_{n}\right)}\right)} \\
& \sup _{\left\|S_{i}^{h} U\right\| W_{1, \mu^{2}\left(R_{n}\right) \leq 1}}\left|A\left(S_{i}^{h} U, S_{i}^{h} V\right)\right| \geqq C\left\|S_{i}^{h} V\right\|_{W_{1,-N^{2}\left(R_{n}\right)}}
\end{aligned}
$$

and

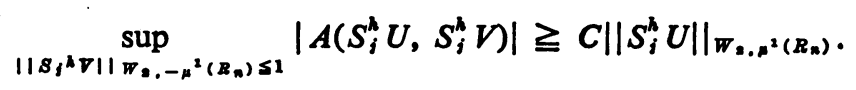

Proof. 1. Inequality (3.9) follows immediately from (3.2).

2. To prove (3.10), take $V \in Q_{-\mu}^{1, \hbar}$ and let $U(k)=V(k) e^{-2 \mu|k| h}$, then

$$
\begin{aligned}
\|U\|_{Q_{\mu^{2}, 1}}^{2} \leqq 2 h^{n} & {\left[\frac{1}{h^{2}} \sum_{k} \sum_{i=1}^{n}\left|V\left(k+1_{i}\right)-V(k)\right|^{2} e^{-2 \mu|k| k}\right.} \\
& \left.+(2 \mu)^{2} C \sum_{k}|V(k)|^{2} e^{-2 \mu|k| h}+\sum_{k}|V(k)|^{2} e^{-2 \mu|k| k}\right]
\end{aligned}
$$

which implies that 


$$
\|U\|_{O_{n}, A} \leqq C\|V\|_{Q_{-\infty}, A, A} .
$$

Hence, by Theorem 2.1, we have

$$
\left\|S_{i}^{h} U\right\|_{W_{*, R^{2}\left(R_{n}\right)}} \leqq C\left\|S_{i}^{h} V\right\|_{W_{0 .-n^{2}\left(R_{s}\right)}} .
$$

Furthermore,

$$
\begin{aligned}
&\left|A\left(S_{i}^{h} U, S_{i}^{h} V\right)\right|=\mid \int_{R_{n}} \sum_{i=1}^{n} \\
& \quad\left[\frac{\partial}{\partial x_{i}} \sum_{k} V(k) e^{-2 \mu|k| h} \varphi_{i}\left(\frac{x}{h}-k\right)\right]\left[\frac{\partial}{\partial x_{i}} \sum_{k} V(k) \varphi_{i}\left(\frac{x}{h}-k\right)\right] d x \\
&+\int_{R_{n}}\left[\sum_{k} V(k) e^{-2 \mu|k| h} \varphi_{i}\left(\frac{x}{h}-k\right)\right]\left[\sum_{k} V(k) \varphi_{i}\left(\frac{x}{h}-k\right)\right] d x \mid .
\end{aligned}
$$

However,

$$
\begin{aligned}
\frac{\partial}{\partial x_{i}} \sum_{k} V(k) e^{-2 \mu|k| h} & \varphi_{i}\left(\frac{x}{h}-k\right) \\
= & \sum_{k} \frac{V\left(k+1_{i}\right)-V(k)}{h} \varphi_{i_{i} 0}\left(\frac{x}{h}-k\right) e^{-2 \mu|k| k} \\
& -2 \mu \sum_{k} V\left(k+1_{i}\right) \varphi_{i, i}\left(\frac{x}{h}-k\right) Q_{k} e^{-2 \mu|k| k}
\end{aligned}
$$

with $Q_{k}$ uniformly bounded, independently of $k$. Now we obtain

$$
\left|A\left(S_{i}^{h} U, S_{i}^{h} V\right)\right| \geqq C\left[\|V\|_{Q_{-\mu^{2}, A}}^{2}-2 \mu C\|V\|_{\left.Q_{-\mu^{2}, A}\right]}^{2} \text { with } C>0\right. \text {. }
$$

In fact,

$$
\begin{aligned}
\int_{R_{n}} & {\left[\sum_{k} V(k) e^{-2 \mu|k| \varphi_{i}} \varphi_{i}\left(\frac{x}{h}-k\right)\right]\left[\sum_{k} V(k) \varphi_{i}\left(\frac{x}{h}-k\right)\right] d x } \\
=\int_{R_{n}} & {\left[e^{-2 \mu|x|}\left[\sum_{k} V(k) \varphi_{i}\left(\frac{x}{h}-k\right)\right]^{2}\right.} \\
& \quad\left[\quad\left[\sum_{k} V(k) \varphi_{i}\left(\frac{x}{h}-k\right)\left(e^{-2 \mu|k| k}-e^{-2 \mu|x|}\right)\right]\left[\sum_{k} V(k) \varphi_{i}\left(\frac{x}{h}-k\right)\right]\right] d x .
\end{aligned}
$$

Using Theorem 2.1, we obtain

$$
\begin{aligned}
\int_{R_{n}} & {\left[\sum_{k} V(k) \varphi_{i}\left(\frac{x}{h}-k\right)\left(e^{-2 \mu|k| h}-e^{-2 \mu|x|}\right)\right]\left[\sum_{k} V(k) \varphi_{i}\left(\frac{x}{h}-k\right)\right] d x } \\
& \leqq \int_{R_{n}}\left[\sum_{k}|V(k)| \varphi_{i}\left(\frac{x}{h}-k\right)|Q(\mu, x, k)| 2 \mu\right]\left[\sum_{k}|V(k)| \varphi_{i}\left(\frac{x}{h}-k\right)\right] e^{-2 \mu|x|} d x \\
& \leqq 2 \mu C\|V\|_{Q-\mu^{2}, h}^{2},
\end{aligned}
$$

where $Q(\mu, x, k)$ is bounded independently of $k$ because of the compact support of $\varphi_{i}(x)$. By a similar argument, using (3.15), we obtain

$$
\begin{aligned}
\int_{R_{n}}\left[\frac{\partial}{\partial x_{i}} \sum_{k} V(k) e^{-2 \mu|k| h} \varphi_{i}\left(\frac{x}{h}-k\right)\right]\left[\frac{\partial}{\partial x_{i}} \sum_{k} V(k) \varphi_{i}\left(\frac{x}{h}-k\right)\right] d x \\
=\int_{R_{n}} e^{-2 \mu|x|} \sum_{i=1}^{n}\left[\frac{\partial}{\partial x_{i}} \sum_{k} V(k) \varphi_{i}\left(\frac{x}{h}-k\right)\right]^{2} d x+R
\end{aligned}
$$


with $|R| \leqq 2 \mu C\|V\|_{Q-\mu^{2}+A .}^{2}$ Hence, for sufficiently small $|\mu|$, we have

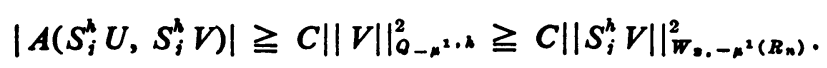

From (3.18), using (3.13), we get-inequality (3.10). Inequality (3.11) is proved by changing $\mu$ to $-\mu$ in the above discussion.

This completes the proof of Theorem 3.2.

4. The Finite Element Method and its Convergence. In [6] we proved the following two theorems.

THEOREM 4.1. Let $H_{1}$ and $H_{2}$ be two Hilbert spaces with scalar product $(\cdot, \cdot)_{H_{2}}$ and $(\cdot, \cdot)_{H,}$, respectively.

Let $B(u, v), u \in H_{1}, v \in H_{2}$, be a bilinear form on $H_{1} \times H_{2}$ such that

$$
\begin{aligned}
|B(u, v)| & \leqq C_{1}\|u\|_{H_{1}}\|v\|_{H_{2}}, \\
\sup _{\|u\|_{H_{2} \leq 1}}|B(u, v)| & \geqq C_{2}\|v\|_{H_{3},}
\end{aligned}
$$

and

$$
\sup _{\|,\| \|, \leq 1}|B(u, v)| \geqq C_{3}\|u\|_{B_{1}},
$$

with $C_{1}<\infty, C_{2}>0, C_{3}>0$.

Let $H_{2}^{\prime}$ be the space of bounded linear functionals on $H_{2}$. Let $f \in H_{2}^{\prime}$. Then there exists exactly one element $u_{0} \in H_{1}$ with

$$
\left\|u_{0}\right\|_{H_{2}} \leqq\|f\|_{H_{1}} / C_{3} \text {, }
$$

such that

$$
B\left(u_{0}, v\right)=f(v)
$$

for all $v \in H_{2}$.

THEOREM 4.2. Let the assumptions of the Theorem 4.1 be fulfilled. Further, let $M$, and $M_{2}$ be closed subspaces of $H_{1}$ and $H_{2}$, respectively. For every $0 \in M_{2}$, let

$$
\sup _{\|u\|_{B_{1} \leq 1}}|B(u, v)| \geqq d_{2}\left(M_{1}, M_{2}\right)\|v\|_{H_{2}}
$$

with $d_{2}\left(M_{1}, M_{2}\right)>0$, and for every $u \in M_{1}$ let

$$
\sup _{\|10\| \|_{B, \leq 1}}|B(u, v)| \geqq d_{3}\left(M_{1}, M_{2}\right)\|u\|_{H_{2}}
$$

with $d_{3}\left(M_{1}, M_{2}\right)>0$.

Let $f \in H_{2}$ be given and let $u_{0}$ denote that element of $H_{1}$ for which

$$
B\left(u_{0}, v\right)=f(v)
$$

holds for all $v \in H_{2}$ (such an element exists and is unique by Theorem 4.1).

Assume there exists $w \in M_{1}$ such that

$$
\left\|u_{0}-w\right\|_{B_{1}} \leqq \vartheta .
$$

Furthermore, let $\hat{u}_{0} \in M_{1}$ such that

$$
B\left(a_{0}, v\right)=f(v)
$$


for all $v \in M_{2}$. Then

$$
\left\|u_{0}-\hat{u}_{0}\right\|_{H_{1}} \leqq\left[1+\frac{C_{1}}{d_{3}\left(M_{1}, M_{2}\right)}\right] \vartheta
$$

We will utilize the Theorems 4.1 and 4.2 to analyze the solution of the equation

$$
-\Delta u+u=f \text {. }
$$

Let us first prove Lemma 4.1.

LEMMA 4.1. Let $f \in W_{2,-\mu}^{0}\left(R_{n}\right)$, then

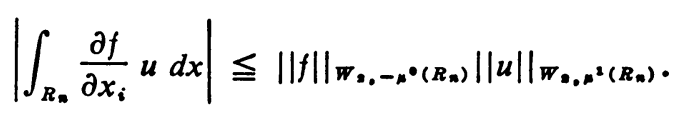

Proof. For any $f \in C^{\infty}$ with compact support,

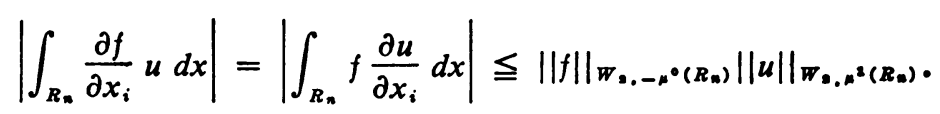

The functions $f \in C^{\infty}$ with compact support are dense in the space $W_{2,-\mu}^{0}\left(R_{n}\right)$, hence, (4.12) holds for all $f \in W_{2,-\mu}^{0}\left(R_{n}\right)$. The following theorem will complete the preparation for the main results of the paper.

THEOREM 4.3. Let $f \in W_{2, \mu}^{l}\left(R_{n}\right), l \geqq 0,|\mu|$ sufficiently small. Then there exists exactly one solution $u$ of $E q$. (1.1) in $W_{2, \mu}^{1}$, such that

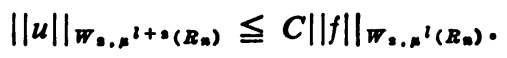

Proof. Since $f \in W_{2, \mu}^{0}\left(R_{n}\right)$, we have

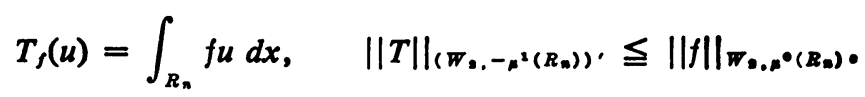

Using Theorems 4.1 and 3.1, we have

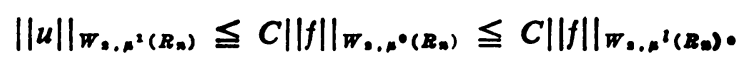

Differentiating both sides of Eq. (1.1), using Theorems 4.1, 3.1 and Lemma 4.1, we obtain

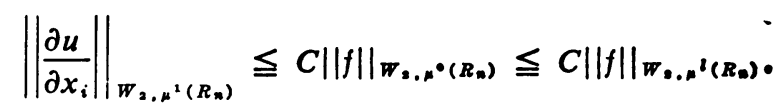

Hence,

$$
\|u\|_{W_{2 . \mu} \cdot\left(R_{n}\right)} \leqq C\|f\|_{W_{2.0^{l}\left(R_{n}\right)}} .
$$

By differentiating Eq. (1.1) $l+1$ times and using induction, we obtain (4.13) for Eq. (1.1).

Let us now describe the finite element method. Let $f \in W_{2,0}^{l}\left(R_{n}\right)$ and let $\psi(h)$ be a decreasing function of $h$ defined for $h>0$. Let

$$
u_{h, \psi, i}(x)=\sum_{|k| \leq \psi(h)} C(k) \varphi_{i}(x / h-k) .
$$

Let us determine the coefficients $C(k)$ such that 


$$
A\left(u_{h, \psi, i}, v\right)=\int_{R_{n}} f v d x
$$

for all $v$ of the form (4.14).

We can now prove the main theorem of the paper.

THEOREM 4.4. Let $f \in W_{2,0}^{l}\left(R_{n}\right)$ and let $\psi(h)=h^{-\bullet}$ for any given $\epsilon>0$. Let $u_{0}$ be the solution of $(1.1)$ in $W_{2,0}^{1}\left(R_{n}\right)$.

Then on every compact domain $\Omega$,

$$
\left\|u_{0}-u_{h, \psi, i}\right\|_{W_{2}^{2}(\Omega)} \leqq C h^{l+1}\|f\|_{W_{0.0^{l}\left(R_{n}\right)}}
$$

for $j \geqq l+2$, where

$$
\|u\|_{W_{i^{2}(\Omega)}}=\int_{\Omega}\left[\sum_{i=1}^{n}\left(\frac{\partial u}{\partial x_{i}}\right)^{2}+u^{2}\right] d x .
$$

Proof. From Theorem 4.3, we have $u \in W_{2,0}^{l+2}\left(R_{n}\right)$. Therefore, $u_{0} \in W_{2, \mu}^{l+2}\left(R_{n}\right)$ for $\mu \leqq 0$ also. Let us use a cut-off function $\chi(x) \in C^{\infty}$ with $\chi(x)=1$ for $|x| \leqq 1$ and $\chi(x)=0$ for $|x| \geqq 2$. Define $\chi_{h, \psi}(x)=\chi\left((\psi(h))^{-1} 3 x\right)$ and $u_{0, h}(x)=\chi_{h, \psi}(x) u_{0}(x)$. Then

$$
\begin{aligned}
u_{0, h}(x)=0, & \text { for }|x| \geqq \frac{2}{3} \psi(h), \\
u_{0, h}(x)-u_{0}(x)=0, & \text { for }|x| \leqq \frac{1}{3} \psi(h),
\end{aligned}
$$

and

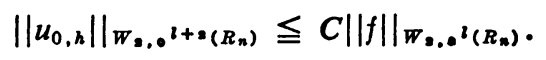

In [14], we have shown that for $j \geqq l+2$ there exist $d(h, k)$ such that

$$
\left\|u_{0, h}-\sum_{k} d(h, k) \varphi_{i}\left(\frac{x}{h}-k\right)\right\|_{W_{1,0^{2}\left(R_{n}\right)}} \leqq C\left\|u_{0, h}\right\|_{W_{1,0^{l+9}\left(R_{n}\right)} h^{l+1}} \text {. }
$$

We have also shown that the support of

$$
w(x)=\sum_{k} d(h, k) \varphi_{i}\left(\frac{x}{h}-k\right)
$$

lies in a $L h$ neighborhood of the support of $u_{0, h}$, i.e. the support of $w(x)$ lies in the set $Q_{h, \downarrow}^{*}=\left\{x ;|x| \leqq \frac{2}{3} \psi(h)+L h\right\}$. Therefore, for $h$ small enough,

$$
Q_{h, \psi}=\{x ;|x| \leqq \psi(h)\} \supset Q_{h, \psi}^{*}
$$

For $\mu<0,|\mu|$ sufficiently small, we obviously have

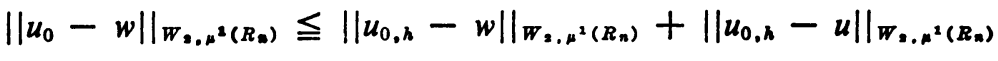

$$
\begin{aligned}
& \leqq C\left[h^{l-1}\|f\|_{W_{1} .0^{l}\left(R_{*}\right)}+e^{\mu \psi(h) / 3}\|f\|_{\left.W_{1 . .0^{l}\left(B_{n}\right)}\right]},\right.
\end{aligned}
$$

since $u_{0, h}-u=0$ for $|x| \leqq \frac{1}{3} \psi(h)$. However, $e^{\mu \psi(h) / 3}=e^{\mu h-\bullet / 3} \leqq C h^{l+1}$. Therefore,

$$
\left\|u_{0}-w\right\|_{W_{2 . N^{2}\left(R_{n}\right)}} \leqq C h^{l+1}\|f\|_{W_{3.0^{2}\left(R_{n}\right)},}
$$

and hence, from Theorems 4.2 and 3.2, we have

$$
\left\|u_{0}-u_{h, \psi, i}\right\|_{W_{1, \mu^{2}\left(R_{n}\right)} \leqq C h^{l+1}}\|f\|_{W_{1,0^{2}\left(R_{n}\right)}},
$$

the desired result. 
Let us count the number $T$ of unknowns in (4.18) which we have to determine by solving a system of linear equations. It is clear that $T$ is of the order $h^{-n(1+\epsilon)}$.

Now let the "effective" $H$ (see [7]) be defined by

$$
H=(T)^{-1 / n} \text {. }
$$

Thus $H=h^{1+\epsilon}$. Hence, instead of (4.16), we may write

$$
\begin{aligned}
\left\|u_{0}-u_{h, \psi, i}\right\|_{W_{\mathbf{a}^{1}(\Omega)}} \leqq C(\epsilon, \Omega) H^{l+1-\epsilon}\|f\|_{W_{2,0^{l}\left(R_{n}\right)}} & =C(\epsilon, \Omega) T^{-(l+1) / n+\epsilon}\|f\|_{W_{2,0^{l}\left(R_{n}\right)}} .
\end{aligned}
$$

Now, by the same manner as in [7], we can show that the rate of convergence indicated in (4.23) is the highest possible rate of convergence on every compact domain, provided that we neglect the $\epsilon$.

Institute for Fluid Dynamics

University of Maryland

College Park, Maryland 20742

1. G. Fix \& G. Strang, "Fourier analysis of the finite element method in Ritz-Galerkin theory," Studies in Appl. Math., v. 48, 1969, pp. 265-273. MR 41 \#2944.

(To appear.)

2. G. STRANG \& G. FIx, "A Fourier analysis of the finite element variational method."

3. G. STRANG, The Finite Element Method and Approximation Theory, Numerical Solution of Partial Differential Equations. II (SYNSPADE, 1970), Academic Press, New York and London, 1971, pp. 547-585.

4. M. Zlámal, "On the finite element method," Numer. Math., v. 12, 1968, pp. 394 409. MR $39 \# 5074$.

5. L. A. OganesJan \& L. A. Ruchovec, "A study of rates of convergence of some variational difference schemes for elliptic equations of second order in a two dimensional domain with smooth boundary," Ž. Vycisl. Mat. i Mat. Fiz., v. 9, 1969, pp. 1102-1119. (Russian)

6. I. BABUŠKA, Error Bounds for Finite Element Method, Technical Note BN-630, Institute for Fluid Dynamics and Appl. Math., University of Maryland, College Park, Md., 1969; Numer. Math., v. 16, 1971, pp. 322-333.

7. I. BABUŠKA, The Rate of Convergence for the Finite Element Method, Technical Note BN-646, Institute for Fluid Dynamics and Appl. Math., University of Maryland, College Park, Md., 1970; SIAM J. Numer. Anal., v. 8, 1971, pp. 304-315.

8. I. BABUŠKA, Finite Element Method for Domains with Corner, Technical Note BN636, Institute for Fluid Dynamics and Appl. Math., University of Maryland, College Park, Md., 1970; Computing, v. 6, 1970, pp. 264-273.

9. I. BABUŠKA, Numerical Solution of Boundary Value Problems by the Perturbed Variation Principle, Technical Note BN-624, Institute for Fluid Dynamics -and Appl. Math., University of Maryland, College Park, Md., 1969.

10. I. BAUŠKA, The Finite Element Method for Elliptic Equations with Discontinuous Coefficients, Technical Note BN-631, Institute for Fluid Dynamics and Appl. Math., University of Maryland, College Park, Md., 1969; Computing, v. 5, 1970, pp. 207-213.

11. I. BABUŠKA, The Finite Element Method for Elliptic Differential Equations, Technical Note BN-653, Institute for Fluid Dynamics and Appl. Math., University of Maryland, College Park, Md., 1970, Numerical Solution of Partial Differential Equations. II (SYNSPADE, 1970), Academic Press, New York and London, 1971, pp. 69-107.

12. O. C. ZIENKIEWICZ, The Finite Element Method in Structural and Continuous Mechanics, McGraw-Hill, New York and London, 1970.

13. Y. R. RASHID, On Computational Methods in Solid Mechanics and Stress Analysis, Conference on Effective Use of Comp. in the Nuclear Industry, Knoxville, Tenn., April 21-23, 1969.

14. I. BabušKa, Approximation by Hill Functions, Technical Note BN-648, Institute for Fluid Dynamics and Appl. Math., University of Maryland, College Park., Md., 1970; Comment. Math. Univ. Carolinae, v. 11, 1970, pp. 787-811. 